\title{
Efecto de ciclofosfamida sobre el metabolismo de los hidratos de carbono en glándula submandibular de ratas
}

\section{Effect of cyclophosphamide on the metabolism of carbohydrates in the submandibular gland of rats}

\author{
Mazzeo MA ${ }^{1}$, Bachmeier $\mathrm{E}^{1}$, López $\mathrm{MM}^{1}$, Linares $\mathrm{JA}^{1}$, Brunotto $\mathrm{M}^{2}$, Dubersarsky $\mathrm{C}^{1}$, Finkelberg $\mathrm{AB}^{1}$ \\ 1. Universidad Nacional de Córdoba. Facultad de Odontología. Cátedra de Fisiología. Argentina \\ 2. Universidad Nacional de Córdoba. Cátedra de Biología Celular "A” INICSA-CONICET. Argentina
}

\begin{abstract}
Objective: During the period of chemotherapy, the appearance of serious systemic toxicities is one of the main clinical problems, among which are those related to the stomatognathic system. Cyclophosphamide is a drug widely used in solid tumors and bone marrow transplantation. The objective of this work was to evaluate the toxicity of cyclophosphamide on the functional activity of the submandibular glands of Wistar rats in an experimental model. Methods: We used 42 male Wistar rats aged 3 months, housed in individual cages, with temperature and controlled lighting and free diet. They were divided into three experimental groups: 1) Control (C), 2) treated with cyclophosphamide (Cf,) applying an i.p. of $50 \mathrm{mg} / \mathrm{Kg}$ of body weight during two consecutive days and 3) rats with paired feeding (AA): during two days the amount administered resulted from the average of the ingested food of group 2 .Both submandibular glands were removed. The submandibular glycogen concentration was analyzed in initial time (t0) and after 60 minutes of mechanical stimulation (t60). Results: The average variations changed significantly between time 0 and 60 in groups $\mathrm{C}$ and AA (p-value $=0.0001$ ), Cf had a lower average concentration in time 0 with respect to $\mathrm{C}$ and AA, without significant consumption in time 60. Conclusion: Cf would affect the metabolism of carbohydrates, decreasing the use of glycogen as a metabolic substrate. In the present experimental model the toxicity of this drug reduces the functional activity of the submandibular gland.
\end{abstract}

KEY WORDS: Wistar rats, cyclophosphamide, glycogen, submandibular gland, toxicity.

\section{Resumen}

Objetivo: Durante el período de quimioterapia, la aparición de toxicidades sistémicas graves es uno de los principales problemas clínicos, entre los que se encuentran los relacionados con el sistema estomatognático. Ciclofosfamida es una droga ampliamente utilizada en tumores sólidos y trasplante de médula ósea. El objetivo de este trabajo fue evaluar la toxicidad de ciclofosfamida sobre la actividad funcional de las glándulas submandibulares de ratas Wistar en un modelo experimental. Métodos: Se utilizaron 42 ratas macho Wistar de tres meses de edad, alojadas en jaulas individuales, con temperatura e iluminación controlada y dieta libre. Fueron divididas en tres grupos experimentales: 1) Control (C), 2) tratadas con ciclofosfamida (Cf) aplicándose una dosis i.p. de $50 \mathrm{mg} / \mathrm{Kg}$ de peso corporal durante dos días consecutivos y 3) ratas con alimentación apareada (AA): durante dos días la cantidad administrada resultó del promedio de la alimentación ingerida del grupo 2 . Se extirparon ambas glándulas submandibulares. Se analizó la concentración de glucógeno submandibular en tiempo inicial ( $\mathrm{t} 0$ ) y luego de 60 minutos de estimulación mecánica (t60). Resultados: Las variaciones promedio cambiaron significativamente entre el tiempo 0 y 60 en los grupos C y AA. (p-valor=0.0001), Cf tuvo una concentración promedio menor en tiempo 0 respecto de $\mathrm{C}$ y AA, sin consumo significativo en tiempo 60. Conclusión: Cf afectaría el metabolismo de los hidratos de carbono, disminuyendo la utilización de glucógeno como sustrato metabólico. En el presente modelo experimental la toxicidad de esta droga reduce la actividad funcional de la glándula submandibular.

PALABRAS CLAVE: ratas Wistar, ciclofosfamida, glucógeno, glándula submandibular, toxicidad. 


\section{Introducción}

Durante el período de quimioterapia, la aparición de toxicidades sistémicas graves es uno de los principales problemas clínicos, entre los que se encuentran los relacionados con el sistema estomatognático ${ }^{1,2}$.

Las complicaciones orales más frecuentes son: mucositis, disgeusia, inflamación, hemorragia gingival y disminución del flujo salival o hiposalivación, un factor que predispone a la xerostomía y otras complicaciones locales ${ }^{3}$.

Ciclofosfamida es una droga ampliamente utilizada asociada a otras drogas oncológicas para el tratamiento de tumores sólidos 0 en esquemas de acondicionamiento para trasplante de médula ósea. Algunos trabajos de otros autores reportaron su toxicidad a nivel clínico y experimental en distintos sistemas orgánicos con alto grado de mitosis $\mathrm{y}$ actividad funcional ${ }^{4,5}$.

El objetivo de este trabajo fue evaluar la actividad funcional de las glándulas submandibulares de ratas Wistar sometidas al tratamiento con el citostático ciclofosfamida a través de la medición de los niveles de glucógeno glandular.

\section{Métodos}

La actividad experimental se llevó a cabo en la Cátedra de Fisiología de la Facultad de Odontología de la Universidad Nacional de Córdoba siguiendo el protocolo internacional para cuidado y tratamiento de animales (NIH) y aprobados por el Centro de Bioética en animales de la Facultad de Ciencias Médicas UNC.

Se utilizaron 42 ratas macho Wistar de tres meses de edad, alojadas en jaulas individuales, con temperatura e iluminación controlada y dieta libre. Fueron divididas en tres grupos experimentales: 1) Control (C), 2) tratadas con ciclofosfamida (Cf) aplicándose una dosis i.p. de $50 \mathrm{mg} / \mathrm{Kg}$ de peso corporal durante dos días consecutivos y 3) ratas con alimentación apareada (AA): durante dos días la cantidad de alimento administrado resultó del promedio de la alimentación ingerida del grupo 2 . El grupo
AA tuvo por finalidad descartar el efecto de la ingesta reducida por acción del tratamiento con $\mathrm{Cf}$ sobre la actividad funcional de las glándulas salivales. Los animales fueron ayunados durante 24 horas previas al sacrificio y anestesiados con una inyección conjunta de Ketamina y Xylazina de 80 y $12,8 \mathrm{mg} / \mathrm{Kg}$ de peso corporal. (Tablas 1 y 2).

Posteriormente se extirparon ambas glándulas submandibulares. La eutanasia de los animales fue practicada mediante dislocación cervical.

Tabla 1. Esquema experimental

\begin{tabular}{|l|l|l|l|l|}
\hline $\begin{array}{l}\text { Grupo } \\
\text { experimental }\end{array}$ & Día 01 & Día 02 & $\begin{array}{l}\text { Día } \\
\mathbf{0 3}\end{array}$ & Día 04 \\
\hline $\begin{array}{l}\text { CONTROLES } \\
\text { n: } 14\end{array}$ & $\begin{array}{l}\text { Sin } \\
\text { tratamiento }\end{array}$ & $\begin{array}{l}\text { Sin } \\
\text { tratamiento }\end{array}$ & Ayuno & Experimento \\
\hline $\begin{array}{l}\text { CF } \\
\text { n: } 14\end{array}$ & $\begin{array}{l}\text { I.P. } \\
\mathrm{mg} / \mathrm{kg} \\
\text { peso } \\
\text { corporal }\end{array}$ & $\begin{array}{l}\text { I.P. } \\
\mathrm{mg} / \mathrm{kg} \\
\text { peso } \\
\text { corporal }\end{array}$ & Ayuno & Experimento \\
\hline AA & $\begin{array}{l}\text { Sin } \\
\text { tratamiento } 14\end{array}$ & $\begin{array}{l}\text { Sin } \\
\text { tratamiento }\end{array}$ & Ayuno & Experimento \\
\hline
\end{tabular}

Tabla 2. Promedio de ingesta diaria de los diferentes grupos experimentales

\begin{tabular}{|c|c|c|c|}
\hline $\begin{array}{c}\text { Día de } \\
\text { tratamiento }\end{array}$ & $\begin{array}{c}\text { Alimentación } \\
\text { ingerida } \\
\text { Grupo control }\end{array}$ & $\begin{array}{c}\text { Alimentación } \\
\text { ingerida } \\
\text { Grupo Cf }\end{array}$ & $\begin{array}{c}\text { Alimentación } \\
\text { apareada }\end{array}$ \\
\hline 1 & $40 \mathrm{~g}$ & $22 \mathrm{~g}$ & $20.5 \mathrm{~g}$ \\
\hline 2 & $41 \mathrm{~g}$ & $19 \mathrm{~g}$ & $20.5 \mathrm{~g}$ \\
\hline 3 & ayuno & ayuno & ayuno \\
\hline
\end{tabular}

Los niveles de glucógeno de la glándula submandibular se evaluaron inmediatamente después de sacrificado el animal (tiempo 0) y luego de 60 minutos de estimulación mecánica en un medio de incubación (KRB) en un medio libre de glucosa siguiendo la técnica de Johann y Lentini ${ }^{6}$.

\section{Análisis estadístico}

La descripción estadística de los datos se realizó mediante su media \pm desvío estándar. La comparación de tratamientos al tiempo 0 y 60 se realizó mediante $\mathrm{t}$ de Student para muestras independientes; 
mientras que la comparación de las variaciones temporales dentro de cada tratamiento se evalúo con la prueba $\mathrm{t}$ de Student para muestras apareadas. En todos los casos se estableció un p-valor $<0.05$ para significación estadística.

Los datos se analizaron con el programa Infostat versión profesional 2018.

\section{Resultados}

Ratas C tiempo 0 mostraron una concentración inicial de glucógeno de 47,46 $\pm 0,9 \mu \mathrm{mol} / \mathrm{g}^{-1}$ p.s (peso seco) que disminuyó en tiempo 60 a 9,18 \pm 0.78 $\mu \mathrm{mol} / \mathrm{g}^{-1}$ p.s. provocando un consumo del $80.66 \%$ del total de los depósitos de glucógeno. Ratas tratadas con alimentación apareada mostraron una concentración inicial de 50,3 $\pm 0.85 \mu \mathrm{mol} / \mathrm{g}{ }^{-1} \mathrm{p} . \mathrm{s}$ que disminuyó en tiempo 60 a $11,5 \pm 0.96$ $\mu \mathrm{mol} / \mathrm{g}{ }^{-1}$ p.s. provocando un consumo del $77.14 \%$ del total de los depósitos de glucógeno

El grupo Cf mostró en tiempo 0 una concentración inicial media de 23,97 $\pm 0,75$ $\mu \mathrm{mol} / \mathrm{g}^{-1}$ p.s que se redujo en tiempo $60 \mathrm{a}$ $20,68 \pm 0.68 \mu \mathrm{mol} / \mathrm{g}^{-1} \mathrm{p} . \mathrm{s}$, implicando un consumo del $13.73 \%$ de los depósitos (Fig. 1)

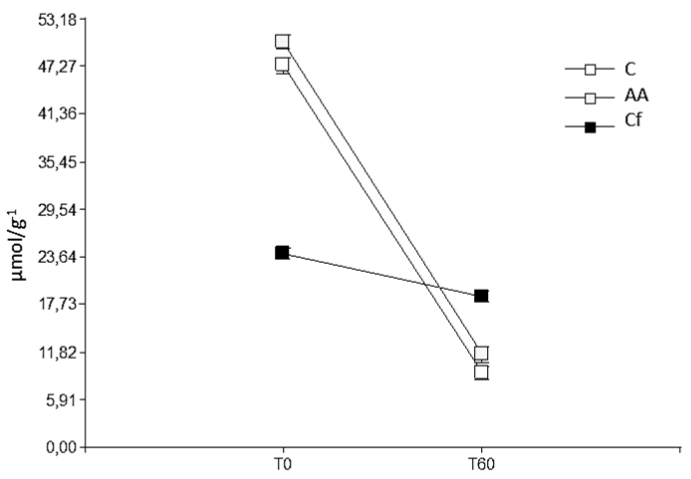

Figura 1. Variaciones promedio en los tratamientos C (control), AA (alimentación apareada), Cf (ciclofosfamida). Las variaciones promedio cambiaron significativamente entre el tiempo 0 y 60 en los grupos C y AA. (p-valor=0.0001, Prueba $t$ Student para muestras apareadas). Cf tuvo una concentración promedio menor en tiempo 0 respecto de C y AA, sin consumo significativo en tiempo 60. $\mathrm{T} 0=$ tiempo cero; $\mathrm{T} 60=$ tiempo 60 minutos .

\section{Discusión}

En condiciones fisiológicas los procesos de secreción salival requieren del aporte de hidratos de carbono como sustrato metabólico. Varios investigadores han establecido la dependencia de las glándulas submandibulares de los mecanismos de glucogenólisis y glucólisis como principales fuentes metabólicas para llevar a cabo la secreción salival ${ }^{7}$.

De este modo, las variaciones del metabolismo del glucógeno glandular son utilizadas in vitro como indicador de la actividad funcional de las mismas ${ }^{8}$.

En el presente trabajo la concentración inicial de glucógeno glandular fue significativamente menor en el grupo tratado con $\mathrm{Cf}$ respecto de los grupos control y alimentación apareada. Luego de la estimulación mecánica, las ratas tratadas con Cf no mostraron cambios significativos en los depósitos de glucógeno glandular. Por el contrario los grupos control y alimentación apareada mostraron una caída significativa de este sustrato al final del período experimental. Un trabajo previo de nuestro equipo realizado con las drogas 5 Fluorouracilo asociado a Leucovorina cálcica (5-FU + LV) mostró un depósito inicial mayor de glucógeno respecto de los grupos control y alimentación apareada, aunque al finalizar el período experimental los resultados fueron similares a los del presente trabajo. No obstante, podríamos hipotetizar que la toxicidad de Cf sobre la actividad funcional de la glándula submandibular sería superior a la observada en ratas tratadas con $5-\mathrm{FU}+\mathrm{LV}^{9}$.

A partir de este resultado podríamos inferir que Cf afectaría el metabolismo de los hidratos de carbono, disminuyendo la utilización de glucógeno como sustrato metabólico en las glándulas salivales.

\section{Agradecimientos}

Agradecemos a la Secretaría de Ciencia y Técnica de la Universidad Nacional de Córdoba (SeCyT) por el financiamiento del presente proyecto de investigación.

Todos los autores declaran que no existen conflictos potenciales de interés con respecto a la autoría y/o publicación de este artículo. 
All authors declare that there are no potential conflicts of interest with respect to the authorship and / or publication of this article

\section{Referencias}

1. Köhne CH, Wils J, Lorenz M, Schöffski P, Voigtmann R, Bokemeyer C, Lutz M, Kleeberg C, Ridwelski K, Souchon R, El-Serafi M, Weiss U, Bukhard O, Ruckle H, Lichnitser M, Langenbuch T, Scheithauer W, Baron B, Couvreur ML, SchmollHJ .Randomized phase III study of high-dose fluorouracil given as a weekly 24-hour infusion with or without leucovorin versus bolus fluorouracil plus leucovorin in advanced colorectal cancer. J Clin Oncol. 2003; 21: 3721-3728.

2. Mazzeo M, Linares JA, Campos ML, Busamia B, Dubersarsky C, Lavarda M, Jarchum G, Finkelberg AB. Oral signs of intravenous chemotherapy with 5-fluorouracil and leucovorin calcium in colon cancer treatment. Med Oral Patol Oral Cir Bucal 2009;14:108113.

3. Bachmeier E, Mazzeo MA, López MM, Linares JA, Jarchum G, Wietz FM, Finkelberg AB. Mucositis and salivary antioxidants in patients undergoing bone marrow transplantation (BMT). Med Oral Patol Oral Cir Bucal. 2014;doi:10.4317/medoral.19062.

4. Martin M, Lluch A, Seguí MA, Ruiz A, Ramos M, Adrover E. et al. Toxicity and health related quality of life in breast cancer patients receiving adjuvant docetaxel, doxorubicin and cyclophosphamide (TAC) or 5-fluorouracil, doxorubicin and cyclophosphamide (FAC): impact of adding primary prophylactic granulocyte-colony stimulating factor to the TAC regimen. Ann Oncol.2006; 17(8) 1205-12.
5. Sonis ST. Oral mucositis in cancer therapy. J Support Oncol 2004; 2 (6 suppl 3): 3-8.

6. Johann C, Lentini EA . Simultaneous determination of glycogen and lipids from heart muscle. Anal Biochem. 1971; 46:186-187.

7. Nicolau J, de Souza DN, Martins HR. Pilocarpine-induced increases in the activity of 6 phosphofructo-2-kinase and the fructose-2, 6bisphosphate content of rat salivary glands. Archs Oral Biol.1992; 37: 483-487.

8. Lanfri-Aguilar $\mathrm{N}$, Gallará $\mathrm{R}$, Linares JA, Campos L, Finkelberg A. Restricted diet modifies carbohydrate metabolism in immature rats. ProstaglandinsLeukotEssentFattyAcids. 2006; $75: 351-355$.

9. Mazzeo MA, Linares JA, López MM, Gallará RV, Bachmeier E, Wietz FM, Finkelberg AB. Functional Impairment in submandibular gland of rats induced by 5-Fluorouracil and calcium leucovorin. Acta Odontol. Latinoam. 2012; 25 (3) :262-68.

Correspondencia a /Corresponding to: Prof. Dr. Marcelo Adrián Mazzeo Cátedra de Fisiología. Facultad de Odontología. $U N C$

Ciudad Universitaria. Córdoba. Argentina. Haya de la Torre s/n. Pabellón Argentina. Correo electrónicole-mail : marceloadrianmazzeo@yahoo.com.ar 